\title{
All-Optical Pulse Rate Multiplication Using Fractional Talbot Effect and Field-to-Intensity Conversion With Cross-Gain Modulation
}

\author{
Shimie Atkins and Baruch Fischer
}

\begin{abstract}
The authors show a passive all-optical method for pulse-rate multiplication. It is based on the fractional temporal Talbot effect along with nonlinear field-to-intensity conversion, using cross gain modulation in a semiconductor optical amplifier. In the experimental demonstration, the pulse rate was doubled and quadrupled to provide $\sim 20-\mathbf{4 0 - G H z}$ pulses, but much higher rates can be obtained with this method.
\end{abstract}

Index Terms-Nonlinear wave propagation, optical fiber communications, optical fiber dispersion, optical pulses, propagation, pulse modulation.

$\mathbf{S}$ IMPLE methods for generating pulses with high repetition rates have great importance in optical communications. At ultra-high rates, where the direct electronical techniques are limited, all-optical methods can be of interest. A recently proposed method using the fractional temporal Talbot effect allows a pseudo-rate multiplication [1], [2], [6] of a given pulse train. However, only the intensity profile is multiplied, which in many cases is sufficient, while the period of the complex field and the spectrum do not change.

In this letter, we show a passive all-optical real-pulse field-rate multiplication method. The method is based on the fractional Talbot effect along with nonlinear field to intensity conversion. The nonlinear part converts the pseudo-rate Talbot multiplication into real rate multiplication. We used for this conversion cross gain modulation (XGM) in a semiconductor optical amplifier. In the present experimental demonstration, a pulse repetition rate that was doubled and quadrupled reached $\sim 40 \mathrm{GHz}$. Nevertheless, much higher rates are achievable. A real rate multiplication can have advantages over a pseudo-multiplication in various cases, especially in propagation of pulses in dispersive lines with such sources. The high pulse repetition rate pulse source presented here allows transmissions of larger information capacity.

In the temporal Talbot effect, a field envelope of an input periodic pulse train with a repetition rate $f$ reproduces itself exactly as it propagates at distances which are multiples of the Talbot length $z_{T}=4 \pi /\left(\left|\beta_{2}\right| \Omega^{2}\right)$ [3], [4], where $\Omega=2 \pi f$ and $\beta_{2}$ is the group velocity dispersion. At half-Talbot distances (odd multiples of $z_{T} / 2$ ), the periodic pulse train is still reproduced, but

Manuscript received April 1, 2002; revised August 20, 2002. This work was supported in part by the Division for Research Funds of the Israel Ministry of Science and by the Fund for Promotion of Research at the Technion.

The authors are with the Department of Electrical Engineering, TechnionIsrael Institute of Technology, Haifa 32000, Israel (e-mail: fischer@ee.technion.ac.il).

Digital Object Identifier 10.1109/LPT.2002.804662 with a shift of half a period. At other distances the pulse train and rate are modified while the shape of each pulse remains the same. For other fractions of the Talbot length, we can obtain corresponding "multiplications" of the repetition rate, where for $z_{T} / 2 m, m$ is an integer; we obtain multiplication of $m$. This multiplication, $m$, is limited by the spectral bandwidth of the original pulse train, which must be broad enough to support the resulting multiplication. However, as was mentioned above, the intensity of the signal is multiplied but not the complex field, since the linear propagation does not change the spectrum or the temporal period of the complex field. This is demonstrated in a numerical simulation of propagation of a pulse train for different fractions of the Talbot length shown in Fig. 1.

Counterpropagating XGM [5] as used in our experiment is shown schematically in Fig. 2. Here, the signal is a pulse train, which has undergone pseudo-repetition rate multiplication, of wavelength $\lambda_{s}$, and is inserted into one side of the semiconductor optical amplifier (SOA), while a continuous wave (CW) signal, of wavelength $\lambda_{c}$, is inserted into the other side. The modulated signal, assuming adequate intensity, causes saturation of the amplifier's gain, thereby modulating the gain, which the relatively weak $\mathrm{CW}$ signal receives, and in effect modulating the CW signal. The output of the amplifier, of wavelength $\lambda_{c}$, now has the complementary format of the original signal, of wavelength $\lambda_{s}$. The original format of the signal can also be obtained either by using a second stage of the XGM, with a semiconductor optical amplifier, or a saturable absorber element instead of the saturable gain. This can be accomplished with the same amplifier, operating in the absorption regime. In any case, the repetition rate of the output signal after conversion is received according to the rate of the intensity of the input signal, where the phase of the input signal is eliminated.

The experimental system is presented in Fig. 3. It consists of a distributed Bragg reflector (DBR) gain-switched laser, generating pulses of $\sim 10 \mathrm{GHz}$ with wavelength $\lambda_{s} \approx 1556.5 \mathrm{~nm}$. For high multiplications of the repetition rate, compression of the pulses was necessary in order to receive enough spectral bandwidth to support the multiplication. This was accomplished using both linear and nonlinear compression in fibers. The linear compression was performed with normal high dispersion fiber while the nonlinear compression was obtained using very high amplification, propagation in standard communication fiber with anomalous dispersion to create a soliton, then attenuation. These pulses were propagated a fraction of Talbot length $z_{T} / 4$ and $z_{T} / 8$, achieving a pseudorepetition rate "doubling" and "quadrupling," respectively. These fractions of the Talbot 

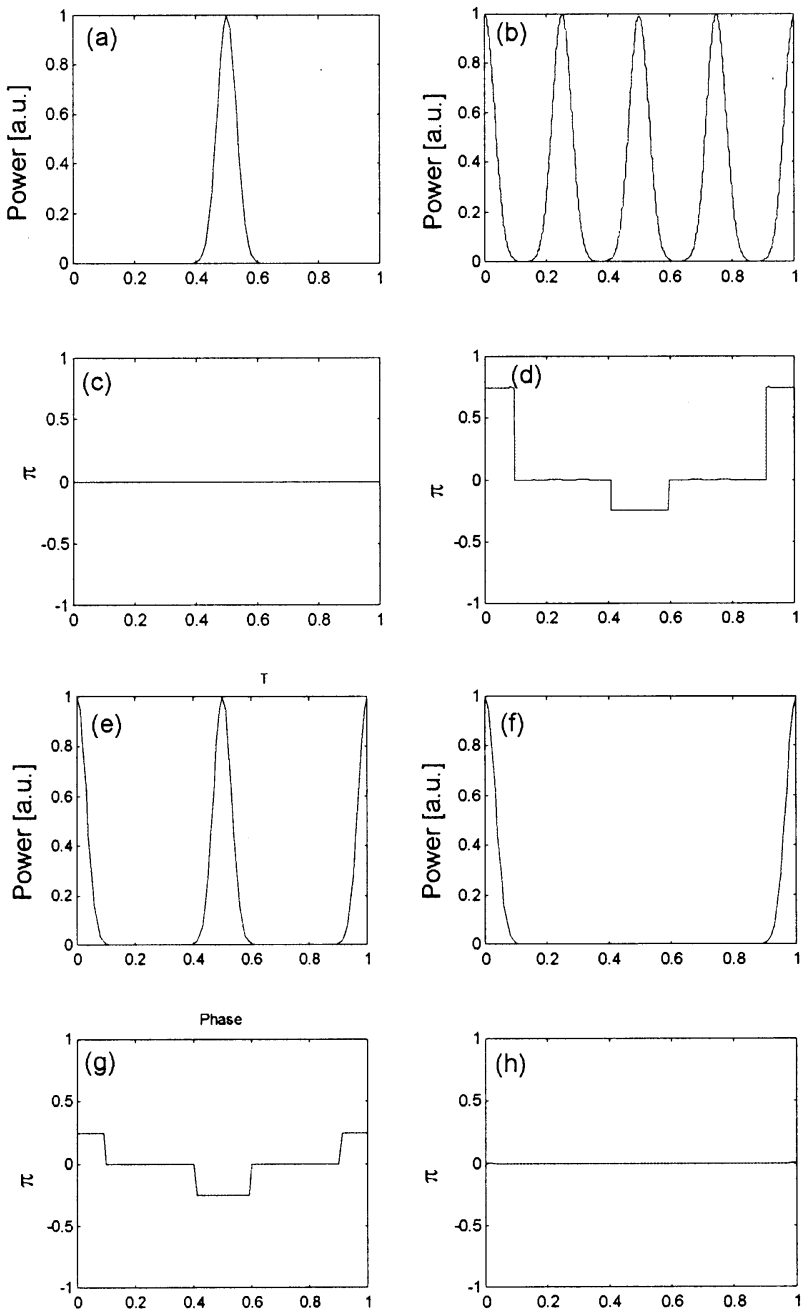

Fig. 1. Simulation of pulse propagation for $z=0$ [(a) intensity and (c) phase], $z=z_{T} / 8,\left[(\mathrm{~b})\right.$ intensity and (d) phase], $z=z_{T} / 4,[(\mathrm{e})$ intensity and $(\mathrm{g})$ phase], and $z=z_{T} / 2$, [(f) intensity and (h) phase], where one pulse cycle is represented and the power is normalized. Note the different phases for the pulses, while the number of pulses is multiplied in the intensity of the complex field.

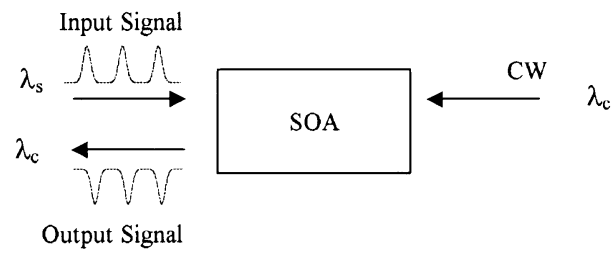

Fig. 2. The schematic of counterpropagating XGM in an SOA.

lengths were obtained using $25 \mathrm{~km}$ of fiber $\left(\beta_{2} \sim-20 \mathrm{ps}^{2} / \mathrm{nm}\right)$ for the former and $3.06 \mathrm{~km}$ of fiber $\left(\beta_{2} \sim+142 \mathrm{ps}^{2} / \mathrm{nm}\right)$ for the latter. The pulses were then amplified and inserted into the semiconductor optical amplifier. The $\mathrm{CW}$ wavelength was approximately $6 \mathrm{~nm}$ lower than the central wavelength of the pulses $\lambda_{c} \approx 1550 \mathrm{~nm}$ so there would be no overlap between the spectrum of the injected pulses and the spectrum of the cross gain modulated pulses. The $\mathrm{CW}$ signal power was $0 \mathrm{dBm}$ for both experiments. The bias current of the amplifier was chosen to be close to the maximum limit, for maximum gain, and was $197 \mathrm{~mA}$ for $z_{T} / 4$ and $176 \mathrm{~mA}$ for $z_{T} / 8$. Figs. 4 and 5 show

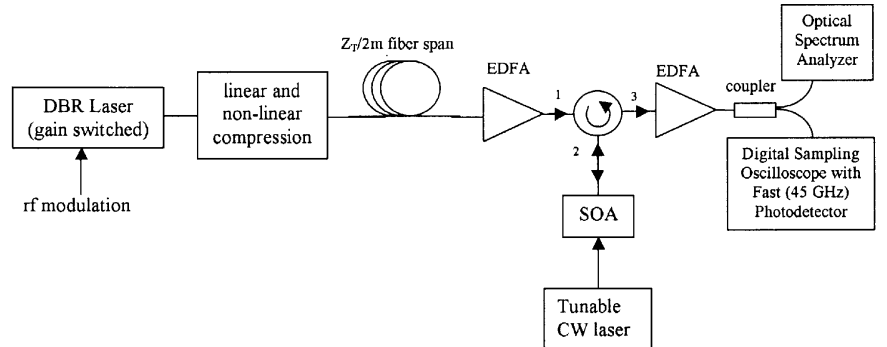

Fig. 3. Experimental configuration of the Talbot frequency multiplier, consisting of a gain-switched distributed Bragg reflector (DBR) laser and a tunable $\mathrm{CW}$ laser, a linear and nonlinear compressor, a long fiber span, erbium-doped fiber amplifiers (EDFA), and an SOA for XGM.
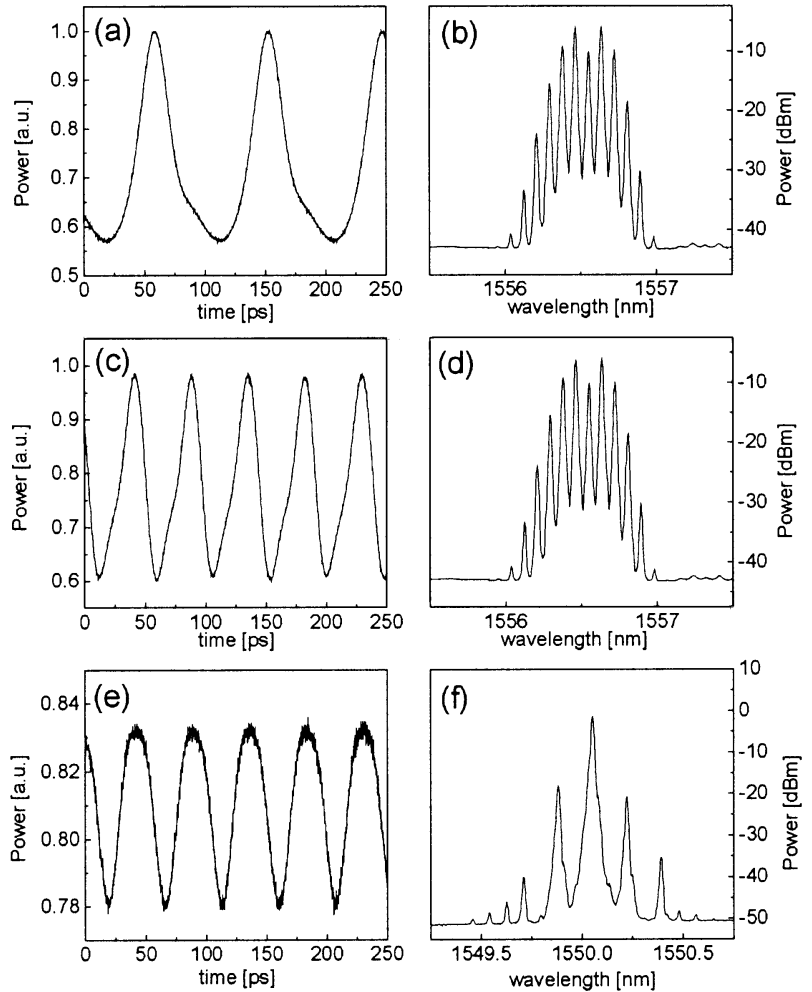

Fig. 4. The pulse train generated by the laser [(a) pulses and (b) spectrum], the pulse train after propagation of $z_{T} / 4$ [(c) pulses and (d) spectrum], and the pulse train after the XGM [(e) pulses and (f) spectrum], demonstrating the frequency multiplication for $f=2 * 10.569 \mathrm{GHz}=21.14 \mathrm{GHz}$, where for $25 \mathrm{~km}$ of anomalous fiber we receive $z_{T} / 4$ (one-quarter of the Talbot length).

the spectrum and pulses of the distributed Bragg reflector laser before "pseudomultiplication" with rates of 10.569 and 9.349 $\mathrm{GHz}$, the spectrum and pulses after propagation of $z_{T} / 4$ and $z_{T} / 8$, respectively (the inputs to the semiconductor optical amplifier) as well as the spectrum and pulses of the amplifier output.

It can be seen from the spectra in Figs. 4(f) and 5(f) that we now receive real repetition rate multiplication. For multiplication by two, every other sideband in the spectrum ceases to exist after the semiconductor optical amplifier, and for multiplication by four only every fourth sideband remains. To achieve higher multiplications the input pulses must have a broad spectrum and the fractional Talbot length must be very precise in order to receive high-quality pulses. If the different pulses in the period 

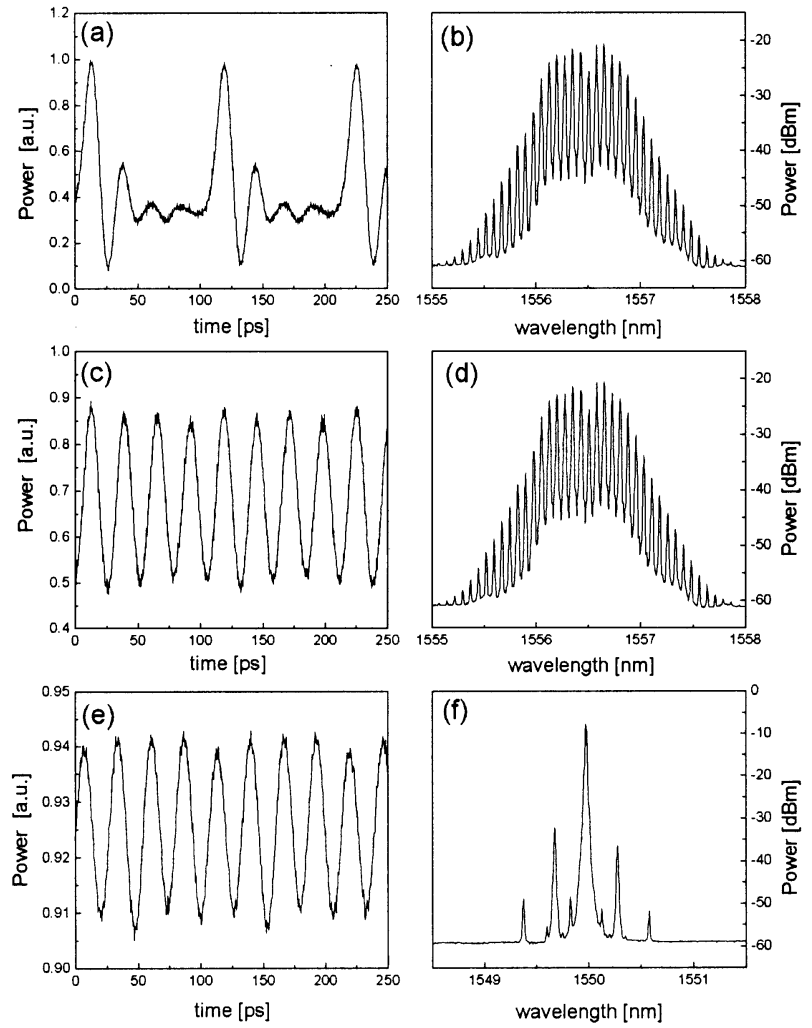

Fig. 5. The pulse train generated by the laser [(a) pulses and (b) spectrum], the pulse train after propagation of $z_{T} / 8$ [(c) pulses and (d) spectrum], and the pulse train after the XGM [(e) pulses and (f) spectrum], demonstrating the frequency multiplication for $f=4 * 9.349 \mathrm{GHz}=37.39 \mathrm{GHz}$ where for $3.06 \mathrm{~km}$ of positive group-velocity dispersion fiber we receive $z_{T} / 8$.

have different intensities, the pulses after the amplifier will have the same differences, corresponding to different repetition rates. Thus in Fig. 5(f), we can see that not all the undesired spectral components were filtered out. In the time domain, due mainly to the low extinction ratio of our semiconductor optical amplifier but also to the addition of the EDFAs, we receive a high dc signal with small modulation; in other words, this process is not highly efficient. We also see a notable degradation of the pulses, where the output pulse train received is essentially the complementary of the input pulse train. These pulses can be improved either by propagating the signal through fiber with positive dispersion, thus negating the high positive chirp at the output of the semiconductor optical amplifier (which causes pulse broadening when propagated through normal fiber) or by performing XGM again with another semiconductor optical amplifier to receive the replicate of the input pulse. The high dc signal can be reduced using several different methods: filtering of the spectral component contributing to the dc, optimization of the output power of the DBR laser, thus lowering the need for amplification with EDFAs, improving the quality of the pulses in the pulse train (reducing the pulse width) and optimization of the XGM used.

In conclusion, we have presented an all-optical passive method for generating pulses with high repetition rates. Experimental demonstration was performed with rate multiplication of a pulse train of $\sim 10 \mathrm{GHz}$ twice and four times, thus generating $\sim 20 \mathrm{GHz}$ and $\sim 40-\mathrm{GHz}$ pulse trains.

\section{ACKNOWLEDGMENT}

The authors acknowledge D. Dahan for his assistance in performing the experiment.

\section{REFERENCES}

[1] J. Azana and M. Muriel, "Technique for multiplying the repetition rates of periodic pulse trains of pulses by means of a temporal self-imaging effect in chirped fiber gratings," Opt. Lett., vol. 24, p. 1672, 1999.

[2] S. Longhi, M. Marano, P. Laporta, O. Svelta, M. Belmonte, B. Agogliati, L. Arcangeli, V. Pruneri, M. N. Zervas, and M. Ibsen, "40-GHz pulsetrain generation at $1.5 \mu \mathrm{m}$ with a chirped fiber grating as a frequency multiplier," Opt. Lett., vol. 25, p. 1481, 2000.

[3] B. Fischer, B. Vodonos, S. Atkins, and A. Bekker, "Dispersion-mode pulsed laser," Opt. Lett., vol. 25, p. 728, 2000.

[4] L. Liu, "Talbot and Lau effects on incident beams of arbitrary wavefront, and their use," Appl. Opt., vol. 28, p. 4668, 1989.

[5] T. Derhuus, B. Mikkelsen, C. Joergensen, S. L. Danielsen, and K. E. Stubkjar, "All-optical wavelength conversion by semiconductor optical amplifiers," J. Lightwave Technol., vol. 14, p. 942, 1996.

[6] J. Azana and M. Muriel, "Temporal self-imaging effects: Theory and application for multiplying pulse repetition rates," J. Select. Topics Quantum Electron., vol. 7, p. 728, 2001. 